\title{
Investigation of Acoustic Emission Caused by Filtration of an Air Flow through a Porous Medium
}

\author{
Yury Zaslavsky, Vladislav Zaslavsky \\ Department of Hydrophysics and Hydroacoustics, Institute of Applied Physics, Nizhny Novgorod, Russia \\ Email: zaslav@hydro.appl.sci-nnov.ru
}

Received September 20, 2011; revised October 8, 2011; accepted November 8, 2011

\begin{abstract}
We study characteristics of acoustic emission caused by an air flow filtered through samples of solid porous pumice either partially filled with glycerin or dry. Some peculiarities of the laboratory setup and the performed experiments are described. Samples of different porosity and several values of the air pressure drop are tested. Physical reasons for the difference in acoustical emission spectra of samples of different porosity and in the relaxation times of the acoustic emission level caused by different porosity and fluid saturation are discussed. It is proposed to use the phenomenon of acoustic emission for detecting the fluid trapped in the pores of cores. The existing results based on the borehole field experiments are rough. The frequency range of signals recorded in these experiments is less than $5 \mathrm{kHz}$ (up to $10 \mathrm{kHz}$ ). It is shown by our laboratory measurements that the required frequency band should be up to $50-60 \mathrm{kHz}$ to reflect the features of the spectral composition of the acoustic emission in predicting the fluid content. The level of acoustic noise under field conditions is much higher, i.e., by $10-15 \mathrm{~dB}$, than the noise achieved under laboratory conditions. Since the sensitivity of the recorders and the frequency range are considerably higher in our experiments, they are unique when applied to the solved problem.
\end{abstract}

Keywords: Acoustic; Emission; Radiation; Porous; Cores; Samples

\section{Introduction}

Acoustic emission generated in many physical processes in solids $[1,2]$ has attracted attention of seismic scientists since the 70 s and was studied in detail in order to find the possibility of using this effect for the prediction of oiland-gas content in entrails of the earth [3,4]. One type of acoustic emission is high-frequency acoustic noise in geological environment that accompanies filtering the fluid or gas in the near-wellbore area and is recorded with geophones installed in the borehole. The problem of studying the acoustic emission produced by filtering the gas through a porous medium has both applied and fundamental aspects. This is evidenced by the conclusions of the works carried out previously by many authors engaged in oil and gas explorations, geophysical surveys in wells [5-9]. The excitation of elastic waves in the skeleton of the medium during gas filtration through a porous material is also theoretically analyzed in ref. [10] using the following model. An air flow penetrates a partially saturated porous medium and squeezes out liquid droplets from contractions of pores to their dilatations. Droplets hold in pore contractions by capillary tension. This model permits to find a qualitative form of the spectrum of elastic waves generated in the skeleton. In this paper, we present results of experimental investigations of acoustic emission, i.e., the air acoustic noise resulting from the interaction of small-scale turbulence of the filtered gas flow and pore walls. The noise is recorded with a microphone (together with noise meter and laptop) in the air near a sample cut of the porous medium through which the air stream flows into the atmosphere. The goal of our analysis is to study the possibility of using this phenomenon for rapid prediction of fluid content in the pore space of test cores extracted from entrails in search and exploration of hydrocarbon deposits. Peculiarities of the acoustic emission will be analyzed to develop new approaches for detecting fluid in pores of the medium lying in the subsoil. In other words, it is required to investigate the influence of the fluid filling the pores of the medium on noise characteristics of acoustic emission by comparing air-filled pores and partially fluid-filled pores. We focus on the spectral content of acoustic noise and the nature of transient processes, such as noise level decrease with a sharp relief of the pressure drop across the sample. These studies have not only applied purposes but may be also helpful in solving fundamental problems of physical acoustics. Thus an analysis of variations in the spectrum of acoustic emission and time of noise decay to the background level, which occur for controlled changes in 
the parameters of the pore space of the medium, will enable one to determine the mechanism of sound generation and localize noise sources arising in the volume of a porous sample as a result of air filtration through it. It is interesting to find out the effect of introducing a certain amount of a viscous fluid, such as hydrocarbons, oil products, etc., into the pores of the medium on the acoustic noise characteristics. Does the mechanism of noise generation that takes place in the case of a "dry" sample remain predominant? It is necessary to have reliable experimental data on the emission characteristics to confirm the known theoretical generation models. A complete analysis, evidently, assumes the implementation of comprehensive studies of variability of these characteristics caused by, e.g., change of pore pressure gradient in the sample, or differences in the nature of the pore tortuosity, a statistical character, and various types of fluids filling the pores. Some results of the initial stage of the research are given below.

\section{Description of Experiment}

A sample of porous pumice is a cylindrical column of diameter $2.5 \mathrm{~cm}$ and length $2.8-3 \mathrm{~cm}$. The sample is pasted inside one end of the outlet hose, the other end of which is connected to an automobile camera with a pressure of 0.3 - $0.4 \mathrm{MPa}$ (controlled by a manometer and restored after each experiment) (see Figure 1). In the open position of the switch, an air flow runs through a sample and goes into the free atmosphere under the action of the differential static pressure drop distributed along the sample. This pressure is controlled by the manometer; its initial value is restored by an electric pump after each measurement. The pressure gauge is used not only for control of the restored initial value of the pressure drop in each experiment but also for reading (every $\sim 1 \mathrm{~s}$ ) of current values of the air static pressure gradient in the sample after the hose is closed by the switch and the relaxation transient process of acoustic emission decrease begins. The maximum rate of the air flow is measured in the range of $10-20 \mathrm{~mm} / \mathrm{s}$ under stationary conditions. Two types of samples are used in the experiments: fine-pore and coarsepore samples. The porosity of the medium in fine-pore samples is $10 \%-20 \%$, while the porosity in coarse-pore samples is $25 \%-30 \%$. To minimize the level of parasitic noise, the $4135 \mathrm{~B} \& \mathrm{~K}$ capacitor microphone and a porous sample with an outlet hose are placed in a soundproof box $(1 \mathrm{~m} \times 1 \mathrm{~m} \times 1 \mathrm{~m})$ with 3 -cm thick polyurethane foam. The box is shown in Figure 1 as the area encircled by a color dashed line. The "zero" acoustic background level inside the box is $40-50 \mathrm{~dB}$. The distance from the end of the sample (outlet hose cut) to the microphone is 2 $3 \mathrm{~cm}$. The Robotron 00024 noise meter connected with a special electric cable to the microphone is used as an amplifier in acoustic measurements. The receiving channel pro- vides a record of the useful signal in the memory of the laptop through Audio Card PCI $7.1\left(f_{\text {strock. }} \sim 92 \mathrm{kHz}\right)$, while the following data processing enables one to carry out the spectral Fourier analysis in a broad frequency band $\Delta f \sim 20 \mathrm{~Hz}-46 \mathrm{kHz}$.

The experiments on recording the acoustic emission are performed in sequence employing the laboratory setup shown in Figure 1. In the first experiment we use a dry sample; in the second experiment the same sample is partially saturated with glycerin, its volume content amounting to $\sim 10 \%-30 \%$ of the pore volume. Glycerin is chosen for the reason of proximity of its characteristics (viscosity and coefficient of surface tension for pore wall wettability) to the same characteristics of crude oil. The measurements are performed with fine-pore and coarse-pore samples. A pair of dry and moistened samples is involved in each experiment. The average pore diameter of the first type sample is $\sim 1.5$ times more than that of the second type sample and the pore concentration of the first type sample is also higher than that of the second one sample, which provides higher permeability of the first type sample to the gas. Since only comparative data are important,

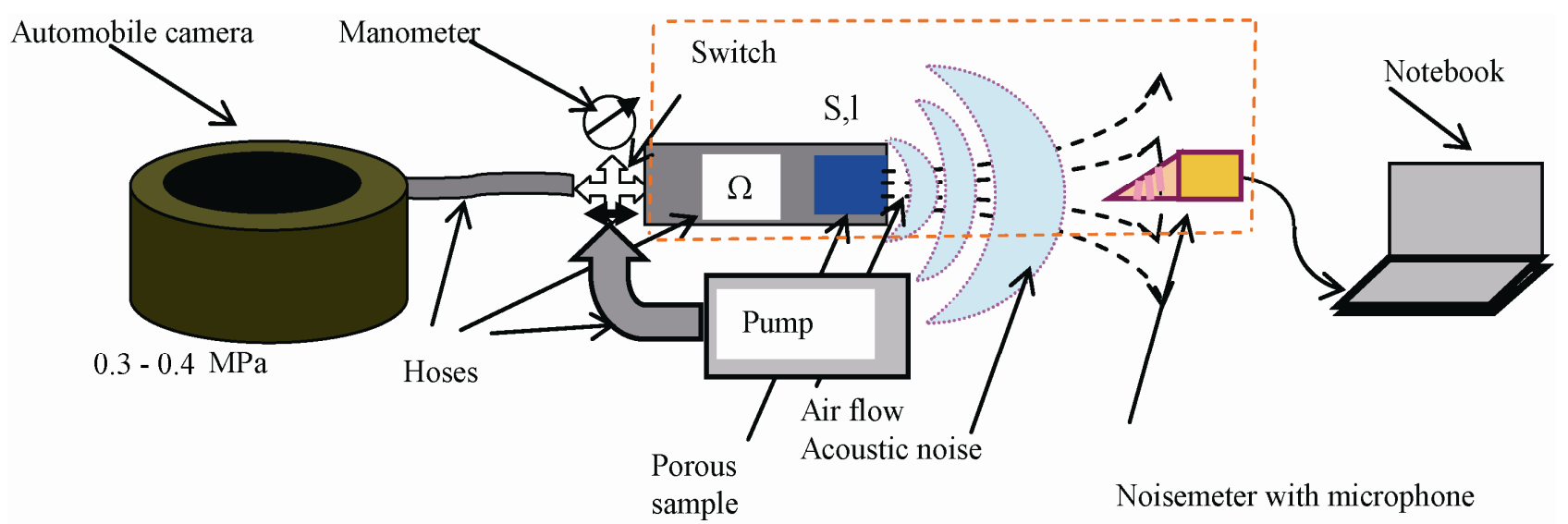

Figure 1. Experimental design. 
we do not present here absolute values of permeability. These values greatly differ from similar parameters of permeability to liquids, which are usually given in literature. Note that the data on the parameters of the tested samples are incomplete, thus the terms "coarse-pore" and "fine-pore" are conventional.

The types of porous media chosen in the experiments are a small fraction of the real variety of species occurring in oil-producing regions. But the differences in quantitative characteristics of the emission arising from the natural spread of the parameters that affect this phenomenon are of interest. Besides the mentioned difference in porosity and wetting, we test two values of the pressure drop across the sample, which allows to determine a qualitative form of the dependence on the value of the specified parameter (in the first case $\Delta p=0.28-0.3 \mathrm{MPa}$ ). To improve the reliability of the results, all experiments and data records are repeated several times.

\section{Discussion of the Results}

Let us consider oscillograms and spectra of acoustic emission recorded near a coarse-pore sample with dry pores at the air pressure drop across the sample equal to $\Delta p=0.3 \mathrm{MPa}$. Relative units are used along the $Y$-axis in oscillograms and spectra of acoustic emission. The oscillogram of acoustic emission including the terminal phase that corresponds to a sharp drop in the air pressure gradient and reduction of the acoustic noise to the acoustic background level after closing the switch and the spectral density of the acoustic emission power are presented in Figures 2(a) and (b). Note that the emission spectrum corresponds to the stationary part of the oscillogram and does not reflect the transient process. It is seen that the acoustic emission level is by $20-30 \mathrm{~dB}$ higher than that of the acoustic background level. The transient process is characterized by the relaxation time and is equal to 2 - 3 s. It follows from Figures 3(a) and (b) that the record of the acoustic emission as a function of time and the measurement data of the air static pressure permit to estimate the threshold pressure $P_{\text {threshould }}$ achieve by a decrease of $P_{\text {static }}$ with respect to $p_{0}$ and corresponding to the decrease of $P_{\text {acoust }}$ down to $P_{\text {background }}$. Simple calculations give the time dependence of the air static pressure in the outlet hose of volume $\Omega$, when air is drained out of it through the porous sample (of cross section $S$ and length $l$ ):

$$
P=p_{\text {atm }} p_{0} /\left(p_{0}-\left(p_{0}-p_{\text {atm }}\right) \exp \left(-S \kappa p_{\text {atm }} t / \Omega \mu_{\text {air }} l\right)\right)
$$

It is seen in the plots of qualitative dependences shown in Figures 3(a) and (b) that the decreases of the pressure levels are exponential in both cases but the time of the static pressure drop significantly exceeds the time of relaxation of the acoustic emission level to the zero acoustic background level. It is easy to estimate the critical

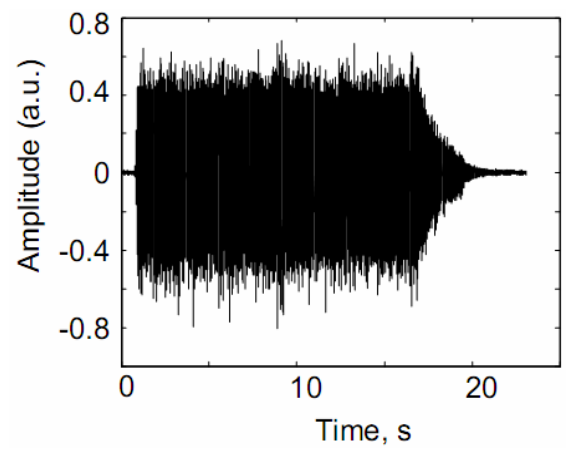

(a)

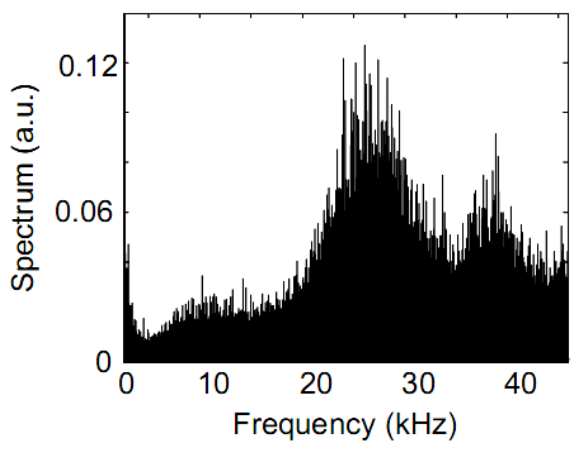

(b)

Figure 2. (a) Oscillogram of acoustic emission (including the relaxation region in the terminal phase); (b) The spectral density of the acoustic emission power, $\Delta p=0.3 \mathrm{MPa}$, the dry coarse-pore sample.



Figure 3. (a) The time dependence of the static pressure drop after closing the switch; (b) A similar time dependence of the acoustic pressure. Level (acoustic emission noise).

value of the gas filtration rate $\left(V_{\text {crit }} \sim P_{\text {threshould }}\right)$ using $P_{\text {threshould }}$ corresponding to the experimentally measured $P_{a c . b a c k g}$ and the known permeability, or vice versa, the permeability using the known critical value of the gas filtration rate. This follows from the dependence that approximately describes gas filtration through the porous sample, which is similar to the Darcy law for a fluid: 


$$
V=\left(\kappa / \mu_{\text {air }}\right) \nabla p
$$

where $\kappa$-the gas permeability, $\mu_{\text {air }}$ - the gas shear viscosity, $\nabla p=\left(P-p_{\text {atm }}\right) / l$ - the pressure gradient, and $l$ - the length of the sample. This dependence is used to derive Equation (1).

Thus taking into account that $\kappa=10^{-13}-10^{-12} \mathrm{~m}^{2}(100-1000 \mathrm{mD})$, where the spread corresponds to different types of samples, $\mu_{\mathrm{air}}=2 \times 10^{-5} \frac{\mathrm{kg}}{\mathrm{ms}}$, $l=3 \times 10^{-2} \mathrm{~m}$ and using the experimentally measured value $P_{\text {threshould }} \approx(1.5-2) \times 10^{5} \frac{\mathrm{kg}}{\mathrm{ms}^{2}}$ and Equation (1), we obtain $V_{\text {crit }}=0.1 \mathrm{~cm} / \mathrm{s}-1 \mathrm{~cm} / \mathrm{s}$. This satisfactorily agrees with the experimental data on filtration rate obtained by video surveillance of a cigarette smoke plume near the outlet hose cut. The time of the transient process when the air static pressure in the sample drops after closing the switch can be estimated by Equation (1). The time of the pressure drop is $\tau=\frac{\Omega \mu_{\text {air }} l}{S \kappa p_{\text {atm }}} \approx 60 \mathrm{~s}$.

The most intense peaks in the noise emission spectrum at stationary air filtration are as follows: the first peak of a higher level is in the frequency range $24-27 \mathrm{kHz}$, while the second peak of a lower level is in the frequency range $36-38 \mathrm{kHz}$. As the pressure drop increases to the value $\Delta p=0.36 \mathrm{MPa}$, the peaks of the spectra shift slightly upward in frequency (by $1-2 \mathrm{kHz}$ ), while the level growth does not exceed $5 \%-10 \%$.

The oscillogram and the spectrum of acoustic emission noise after injection of $0.5 \mathrm{ml}$ of glycerin in the porous of the sample are shown in Figures 4(a) and (b). The relaxation time increases up to $5-6 \mathrm{~s}$. The first maximum in the spectrum has a lower level than the second one and overlaps frequency range $20-30 \mathrm{kHz}$. The second maximum becomes dominant and its frequency band is 35 $39 \mathrm{kHz}$. The same situation takes place both at the air pressure drop $\Delta p=0.36 \mathrm{MPa}$ and at $\Delta p=0.3 \mathrm{MPa}$.

The consideration of similar characteristics of the acoustic emission caused by filtration of an air flow through the fine-pore sample shows that the use of only spectral peculiarities is insufficient to develop the key sign in the search and detection of the fluid present in the pores of such cores. The oscillogram and the spectral density of the acoustic emission power are shown in Figures 5(a) and (b). The initial part of the oscillogram and its spectrum correspond to stationary conditions when the air flow is pumped through the fine-pore dry sample at a pressure drop $\Delta P=0.3 \mathrm{MPa}$. In this case, the acoustic emission noise spectrum has an anomalous broad band with 3 - 4 maxima on the envelope curve even if the pores of the medium are not filled with glycerin. The envelope curve maxima occur at $10 \mathrm{kHz}, 25 \mathrm{kHz}, 35 \mathrm{kHz}$, and

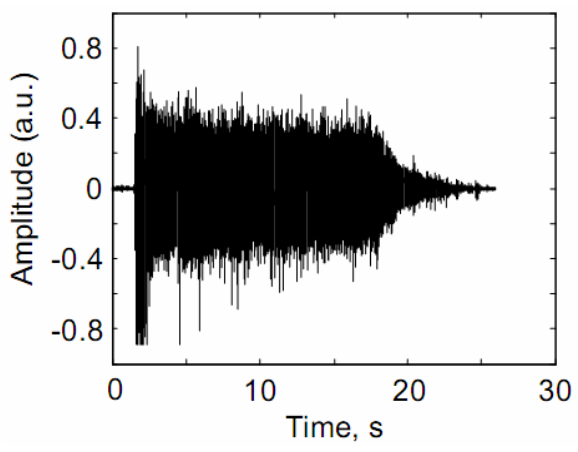

(a)

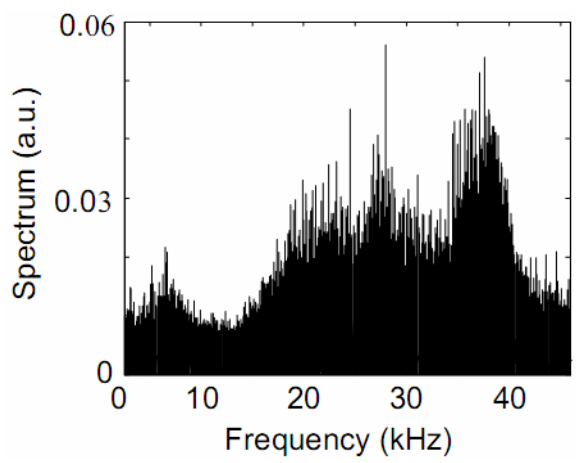

(b)

Figure 4. (a) Oscillogram of acoustic emission; (b) The spectral density of the acoustic emission power. $\Delta p=0.3 \mathrm{MPa}$, the coarse-pore sample filled with $0.5 \mathrm{ml}$ of glycerin.

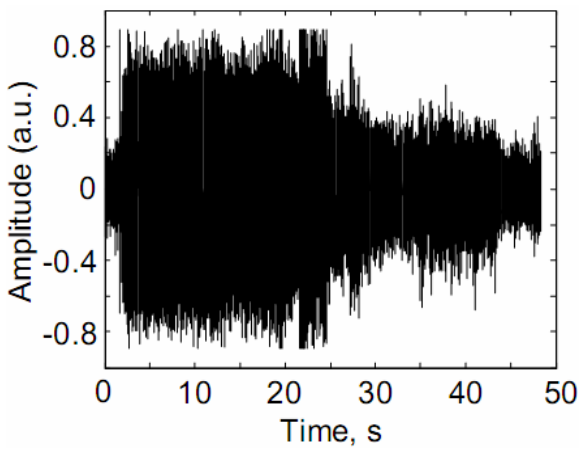

(a)

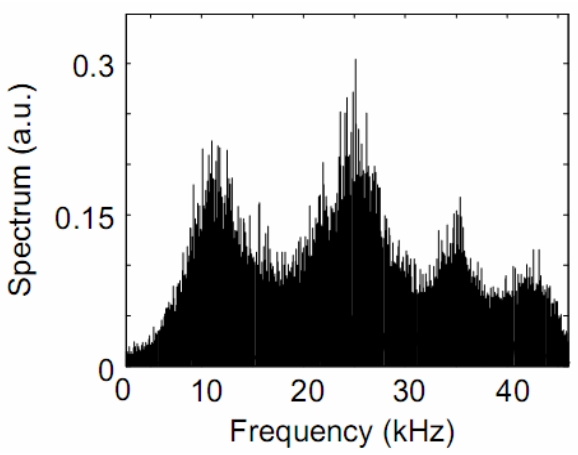

(b)

Figure 5. (a), (b) Oscillogram and the spectrum similar to those shown in Figure 2, the fine-pore dry sample. 
$45 \mathrm{kHz}$. The characteristic time of the noise decay to the background level in the transient process of acoustic emission is $25 \mathrm{~s}$.

The same characteristics of the acoustic emission obtained after injection of $0.5 \mathrm{ml}$ of glycerin in the finepore pumice sample, through which the air flow is filtered at the air pressure drop $\Delta p=0.36 \mathrm{MPa}$ are presented in Figures 6(a) and (b). Only the time interval of $\sim 30 \mathrm{~s}$ is shown in Figure 6(a), while the total relaxation time is up to $\sim 2 \mathrm{~min}$ in this case. The acoustic emission is characterized by a higher integral noise level that exceeds the previous value approximately $1.5-2$ times. A single significant maximum is observed in the spectrum shown in Figure 6(b), which overlaps the frequency band $26-33 \mathrm{kHz}$. A fourfold increase of the transient process time, a higher spectral density of the acoustic emission in the frequency band, i.e. close to the single maximum of the spectral density of the acoustic emission, are the signs of the fluid presence in the sample of this type.

The difference in the relaxation times in the fluid-filled coarse-pore or fine-pore structures and in the air-filled or dry pores can be used as a key sign. This parameter is an important informative sign which together with spectral characteristics can indicate the fluid presence in the pores of the core and hence at the explored productive horizons.

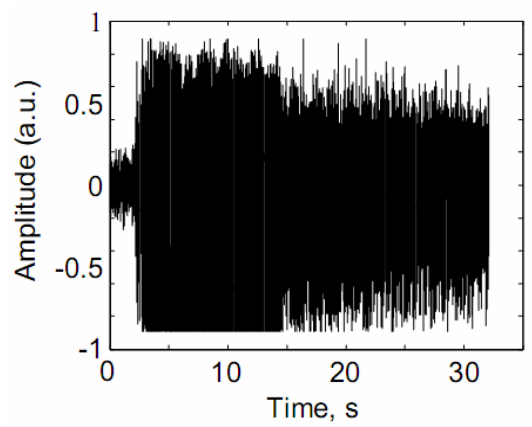

(a)

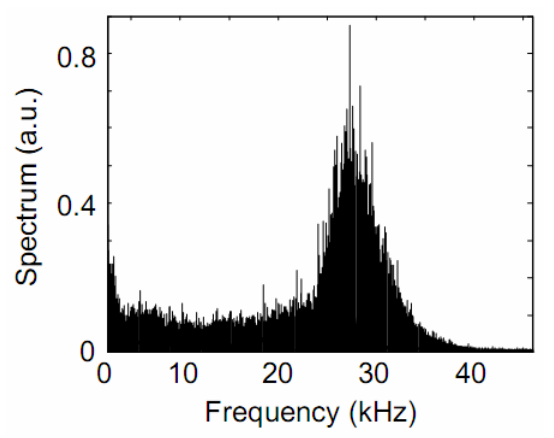

(b)

Figure 6. (a), (b) Oscillogram and the spectrum similar to those shown in Figure 4, the fine-pore sample is filled with $0.5 \mathrm{ml}$ of glycerin.
The following signs of the fluid presence in the core of the porous medium can be proposed due to the results presented above. These are an extension of a frequency band, a higher spectral density power, a higher integral level of the acoustic emission noise in stationary conditions of air filtration through the pores of the skeleton and a higher time of the duration of transitional relaxation process. Made statements have a quality sense only because the experimental data are not enough representative ensemble as yet. The exhaustive determination of the real acoustic emission characteristics by the fluid filling of the porous medium with the use of the obtained results of performed experiments is not possible still and it is required further more detail experiments and careful measurements on the samples with typical entrails structures corresponding to the areas where the exploration of oil deposits is carried out but which don't involved in our experiments. The types of porous rock materials should be broadening further and more wide class of parameters so as density, porosity, permeability are necessary to include in the analysis.

\section{Conclusions}

Key-signs of a fluid filling the pores of the rock materials, based on the phenomenon of acoustic emission noise accompanying an air flow filtered through a porous medium, are proposed. They are obtained as a result of systematizing and processing of laboratory experimental data on acoustic emission characteristics of an air flow filtered through porous pumice samples either with glycerin filling or without it. These key signs can be used to predict the fluid presence in the cores. They are the increase of the acoustic emission level up to 1.5 times with respect to the level of dry sample, the broadening of the frequency band of the acoustic emission spectrum on both sides, and the longer time of emission decrease to the background level (40 - $50 \mathrm{~dB})$.

In the laboratory experiments, we employ two types of porous pumice samples and glycerin which simulate a hydrocarbon accumulation in the entrails of the earth. It is possible to predict the presence or absence of a fluid in a porous material by analyzing the acoustic emission spectra under stationary conditions and to determine the relaxation time of the transient of acoustic decrease down to the background level ( $40-50 \mathrm{~dB})$.

Hence the obtained data are of interest for both the fundamental science research and the oil-gas exploration.

\section{REFERENCES}

[1] V. M. Baranov, "Acoustic Measurements in Nuclear Energy Industry,” Energoizdat, Moscow, 1990.

[2] A. L. Aleinikov, V. T. Belikov and N. I. Nemzorov, "Acoustic Emission in Heterogeneous Media," Defek- 
toskopia, No. 3, 1999, pp. 31-36.

[3] A. L. Aleinikov, V. T. Belikov, N. I. Nemzorov and A. K. Troyanov, "Interpretation of the Observations of the Acoustic Emission Signals on Ural Super Deep Bore Hole," Geology and geophysics, No. 6, 1992, pp. 118126.

[4] L. N. Rykunov, O. B. Khavroshkin and V. V. Tsyplakov, "Time Variations of High Frequency Seismic Noises" Izvestia AN USSR Phyzics of the Earth, No. 11, 1979, pp. 7277.

[5] A. I. Ipatov, A. V. Gorodnov, S. I. Ipatov, N. N. Mar'enko, L. P. Petrov and S. P. Skopintsev, "Investigation of Amplitude-Frequency Signal Spectrums of Acoustic and Electromagnetic Noise by the Fluid Filtration in the Pores," Geophysics, No. 2, 2004, pp. 25-30.

[6] E. F. Afanas'ev, et al., "Control of Deposit Exploration by the Acoustic Method," Review VNIIEgasprom, No. 1, 1987.

[7] Y. P. Korotaev, et al., "Investigation of Gas Wells with the Help of Noise Measurement," Review VNIIEgasprom, No. 1, 1983.

[8] R. M. McKinley, E. M. Bower and R. C. Rumble, "The Structure and Interpretation of Noise from Flow behind Cemented Casing," Journal of Petroleum Technology, Vol. 25, No. 3, 1973, pp. 329-338.

[9] V. N. Nikolaevskiy, "Mechanics of Porous and Cracked Media," Nedra, Moscow, 1984, p. 232.

[10] Y. M. Zaslavsky "On the Theory of Acoustic Emission by Gas Filtration of Partially Fluid Saturated Medium," Electronic Journal “Technical Acoustics”, No. 5, 2005. 